\title{
Ley de Faraday y Ley de Biot y Savart: Campo magnético de una espira circular en un punto fuera del eje
}

Prof. Cintia Cabanillas Mendoza*, Lic. Lucy A. Valdez*, Ing. Cesar A. Cabrera*, Ing. Carlos A. Merino*, Dra. Erika N. Bentz* y Dr. Patricio F. Provasi*

\section{Resumen}

Este trabajo resulta a partir de la indagación bibliográfica relacionada con la medida y el mapeo del campo magnético fuera del eje de una bobina circular tanto en su componente radial como axial, la cual resultase muy escasa.

Se presenta aquí la solución analítica completa, junto con el diseño y la fabricación de un dispositivo para realizar la medición en una región del espacio, de la f.e.m. inducida por un campo magnético generado por una espira circular. El campo magnético se produjo con corriente alterna y la región del espacio considerada comprende un plano donde uno de sus lados contiene al eje de la espira y se extiende más allá de la circunferencia de la misma.

A diferencia de la mayoría de los dispositivos que se encuentran en el mercado y también de los ejemplos que se ven en la bibliografía; el equipo diseñado permite sensar el campo magnético producido por una espira circular en sus inmediaciones tanto en la dirección radial (r) como en la axial (z).

Se han comparado las mediciones experimentales de la f.e.m. inducida con los calculados en ambas direcciones, logrando reproducir satisfactoriamente la forma de los campos magnéticos calculados.

\section{Palabras claves:}

Campo Magnético, Ley de Faraday, Ley de Biot y Savart, Espira Circular.

\section{Abstract}

This work is presented from the bibliographic inquiry related to the measurement and the mapping of the magnetic field outside the axis of a circular coil in both its radial and axial component, which is very scarce.

We present here the complete analyti-

*Departamento de Física, FaCENA - UNNE - 0379-475-0698, patprovasi@gmail.com 
cal solution, in addition to the design and manufacture of a device to perform the measurement, in a region of space, of the induced e.m.f. by a magnetic field generated by a circular loop. The magnetic field is produced with alternating current and the region of space is a plane which includes in one of its sides the axis of the coil and extends itself beyond the circumference of the loop.

Differently to the majority of the devices that are in the market and also of the examples that are seen in the bibliography; the designed equipment allows sensing the magnetic field produced by a circular coil in its vicinity in both, the radial $(r)$ and the axial ( $z$ ) directions.

The experimental measurements of the f.e.m. induced with those calculated in both directions, successfully reproducing the shape of the calculated magnetic fields.

Keywords: Magnetic Field, Faraday's Law, Biot and Savart's Law, Circular Coil.

\section{Introducción}

Existen numerosos experimentos de mapeo y/o medición del campo eléctrico en dos dimensiones, pero el número de éstos decae significativamente cuando se trata del campo magnético, y más aún si pensamos en medir contribuciones mutuamente perpendiculares. De esta forma nuestra principal motivación para realizar el presente trabajo se fundamenta en el escaso número de referencias relacionada con la medida y el mapeo del campo magnético fuera del eje de una bobina circular, tanto en su componente radial como axial.

Normalmente, en la bibliografía correspondiente al grado de física, se trata el problema del campo magnético de la espira en el eje de la misma o bien rápidamente se llega a la aproximación dipolar. Ejemplos de esta índole pueden observarse en las referencias $[1,2,3,4]$.

También es correcto que la solución analítica en todo el espacio es compleja y, en general, escapa al tiempo y al alcance de la asignatura correspondiente, destinados al campo magnético. Sin embargo el mapeo del campo es mucho más sencillo de realizar experimentalmente, siempre que se tomen los recaudos correspondientes.

En este trabajo se presenta la solución analítica completa y el diseño y fabricación de un dispositivo para realizar la medición del campo magnético de una espira en el plano de simetría que contiene al eje de la misma.

\section{Teoría y algo de Historia}

Hans Christian Oersted en 1819, observó que la aguja de una brújula se desviaba ante la presencia de un conductor por el cual circularía una corriente. Poco tiempo después, en 1920, Jean Baptiste Biot y Félix Savart realizaron experimentos sobre los efectos que producía un portador de corriente eléctrica sobre un imán cercano. Se dieron cuenta que si un conductor conduce una corriente constante $\mathrm{I}$, el campo $\mathrm{dB}^{\urcorner}$en 
un punto $\mathrm{P}$ debido a un elemento $\mathrm{dl}^{\rightarrow}$ (ver Figura 1 ) tiene las siguientes propiedades:

1. El vector $d \vec{B}$ es perpendicular tanto a $d \vec{l}$ (el cual tiene la dirección de la corriente) como al vector unitario $\vec{r}$ dirigido desde el elemento hasta el punto $\mathrm{P}$.

2. La magnitud $d \vec{B}$ es proporcional a

a) $r^{2}$, donde $r$ es la distancia desde el elemento $d \vec{l}$ hasta el punto $P$.

b) la corriente I y a la longitud del elemento $d \vec{l}$.

c) $\operatorname{sen} \theta$, donde $\theta$ es el ángulo entre $d \vec{l} \mathrm{y} \vec{r}$.

De esta forma, resulta

$$
d \vec{B}=\frac{\mu_{0}}{4 \pi} \frac{r \cdot d \vec{l} \times r}{r^{2}}=\frac{\mu_{0}}{4 \pi} \frac{i \cdot d \vec{l} \cdot r \operatorname{sen} \theta}{r^{2}}
$$

suponiendo que I es constante, por simplicidad, e integrando sobre toda la distribución de corriente se obtiene $\vec{B}=\frac{\mu_{0} I}{4 \pi} \oint \frac{d \vec{d} \times r}{r^{2}}$

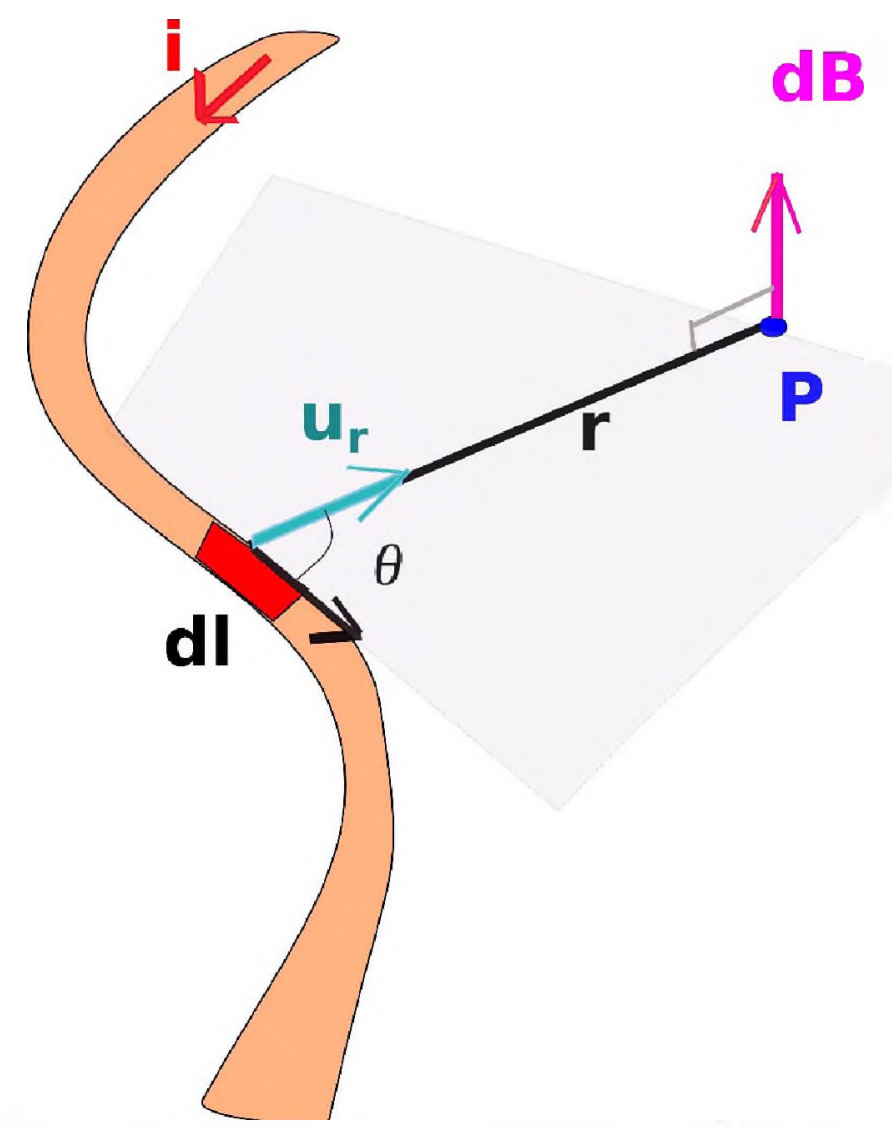

Figura 1: Esquema del campo magnético diferencial d $\overrightarrow{\mathrm{B}}$ producido por un vector de corriente diferencial $I \cdot d l \overrightarrow{ }$ 
Michael Faraday, en 1831, descubrió la inducción electromagnética, esto es, si el flujo magnético que atraviesa una superficie delimitada por una espira conductora varía, se establece sobre dicha espira una corriente eléctrica (detectada por un galvanómetro) que se mantiene mientras el flujo magnético se mantenga variable. Incluso si el circuito está abierto, es decir, no hay circulación de corriente; esa fuerza electromotriz está presente.

Mediante los estudios y experimentos concernientes al flujo magnético, también realizados en forma casi simultánea por Joseph Henry, se demuestra que si el flujo magnético que atraviesa la superficie del circuito varía, se induce una fuerza electromotriz (f.e.m. o $\varepsilon$ ) que es igual en magnitud a la variación por unidad de tiempo del flujo magnético. Es decir: "la f.e.m. inducida en un circuito es igual a la velocidad de variación del flujo magnético" que se conoce como ley de Faraday.

Estudios similares fueron realizados por Frederic Emile Lenz, quien descubrió que "la f.e.m. y la corriente que se inducen en el circuito, tienen un sentido tal que tienden a oponerse a la variación del flujo magnético que las crea", que se conoce como ley de Lenz.

Esto significa que la corriente inducida, al circular, crea un nuevo campo magnético, cuyas líneas se suman a las líneas del campo magnético inductor en sentido y magnitud tal que tienden a contrarrestar la variación del flujo inductor. Consecuentemente, el flujo magnético $\phi$ y la f.e.m. inducida se expresan de la forma

$$
\phi=\iint \vec{B} \cdot d \vec{S}=\iint \vec{B} d \vec{S} \cos \theta \quad \text { f.e.m. }=\varepsilon=-\frac{d \phi}{d t}
$$

esta ultima expresión se conoce como la ley de Faraday-Lenz.

\section{Materiales y Métodos}

Los materiales necesarios para realizar la experiencia fueron:

- Bobinas

- Banco porta bobinas

- Reóstato (Rv)

- Fuente de C.A.

- Voltímetro C.A.

- Amperímetro C.A.

- Cables de conexión

El experimento consiste en hacer circular por una bobina (bobina inductora)
B1 una corriente alterna de forma que ésta genere un campo magnético variable. Se coloca una espira circular $\mathrm{B} 2$ de $\mathrm{N}$ vueltas $\mathrm{y}$ dimensiones conocidas dentro de este campo magnético a una distancia $\mathrm{x}$ de la bobina.

Como el campo magnético varía con respecto al tiempo; las líneas de campo generarán un flujo magnético variable a través de la superficie delimitada por la espira. A1 variar el flujo magnético respecto del tiempo, de acuerdo con la Ley de Faraday, inducirá en la espira B2 una f.e.m. cuya magnitud es igual a la velocidad de variación del flujo. 
La f.e.m. inducida se mide entre los extremos de la espira, y mediante la aplicación de la Ley de Faraday - Lenz se puede determinar la intensidad del campo magnético a la distancia $\mathrm{x}$ de la bobina inductora.

$$
B=-\frac{\varepsilon}{N \cdot \omega \cdot A}
$$

Siendo que se considera que $\boldsymbol{\varepsilon}=$ f.e.m., el campo magnético varía en el tiempo en la forma $B=B_{0} \operatorname{sen}(\omega t), \mathrm{N}$ es el número de espiras de $\mathbf{B}_{2}$ y $\mathbf{A}=\boldsymbol{\pi} \mathbf{R}_{2}^{2}$.

Se repite el procedimiento para distintos puntos y se analiza mediante un gráfico la variación de la intensidad del campo magnético respecto de la distancia $\mathrm{x}$ que separa ambas espiras.

El proceso inverso también podría emplearse, pero no formaba parte del el objetivo de este trabajo y por lo tanto no se realizó. El proceso inverso consiste en calcular mediante la expresión de la Ley de Biot y Savart, el campo magnético en distintos puntos sobre el eje de la bobina inductora. Con estos valores de campo magnético y las dimensiones de la segunda bobina, se calcula, aplicando la Ley de Faraday, el valor de la f.e.m. inducida en cada posición.

Finalmente, los valores de las f.e.m. así calculados, para las distintas posiciones, se comparan con los valores de f.e.m. medidos debiendo verificarse el cumplimiento de las Leyes de Biot y Savart y de Faraday.

En las Figuras 2, 3 y 4 se muestran los esquemas del dispositivo y las configuraciones utilizadas para la experiencia.

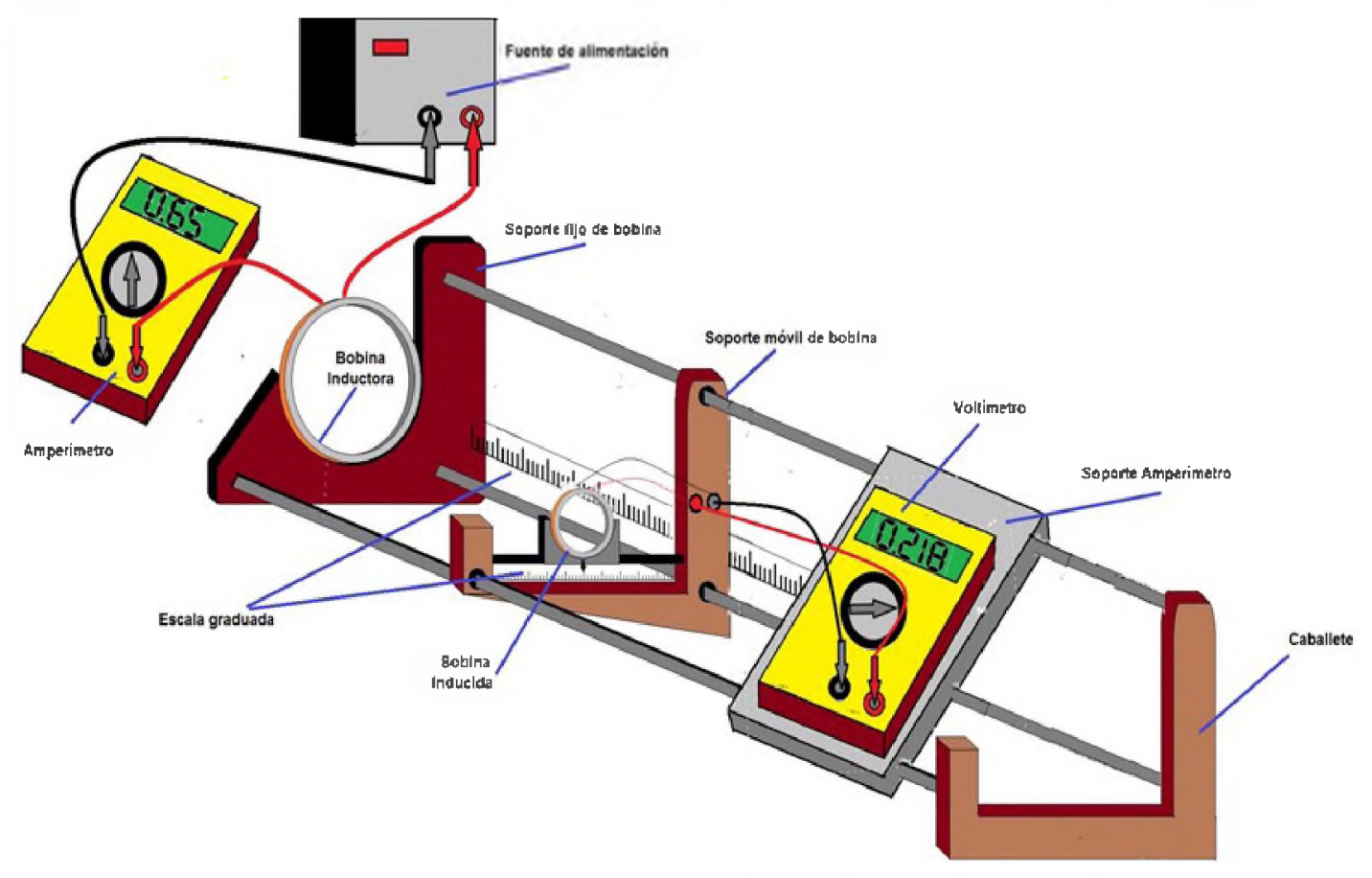

Figura 2: Esquema del dispositivo diseñado para la medición del campo magnético de una espira circular. 

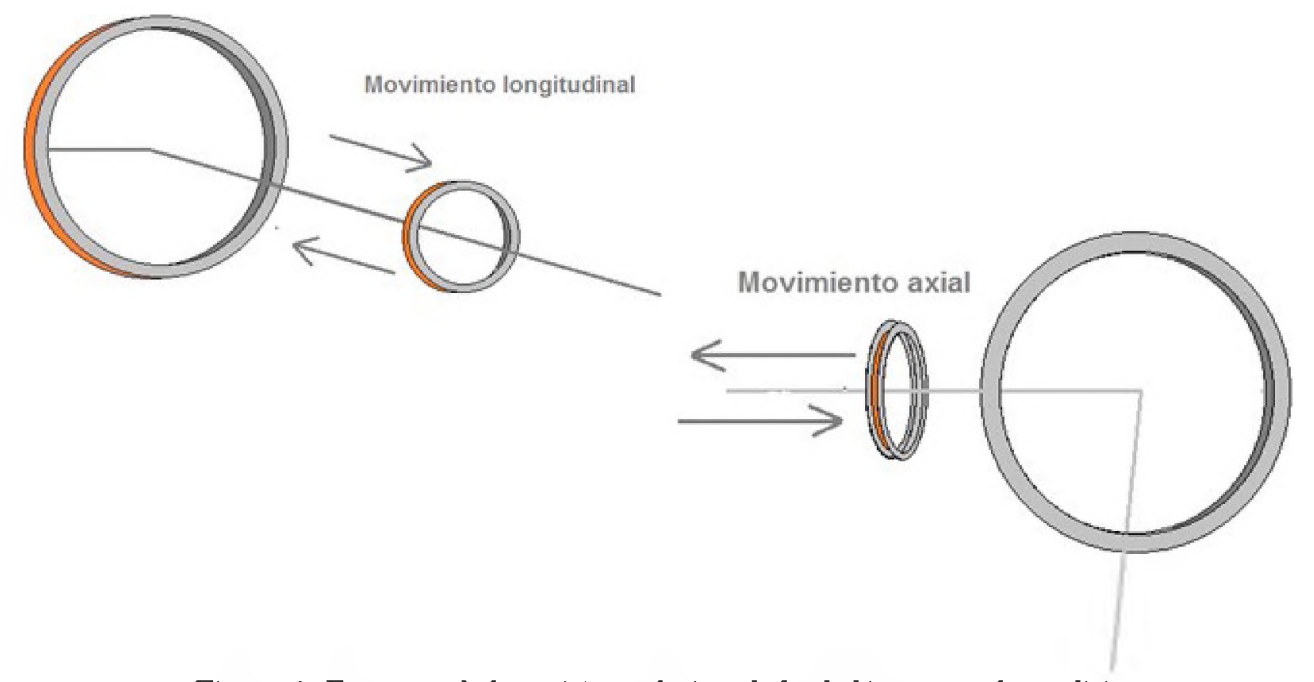

Figura 3: Esquema de la posición relativa de las bobinas para la medición del campo Axial y Radial respectivamente.

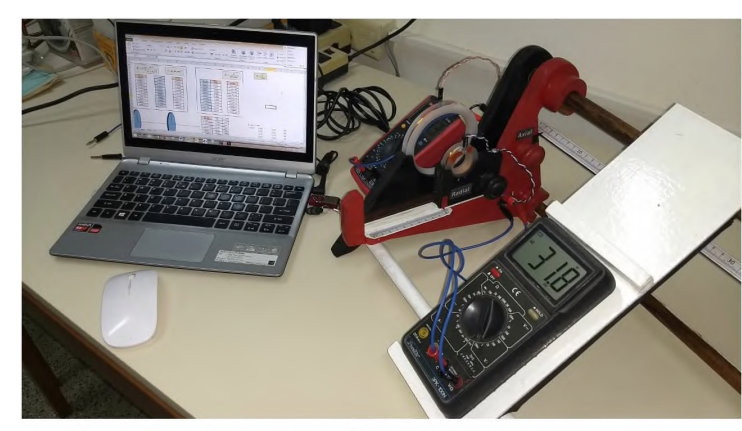

(a)

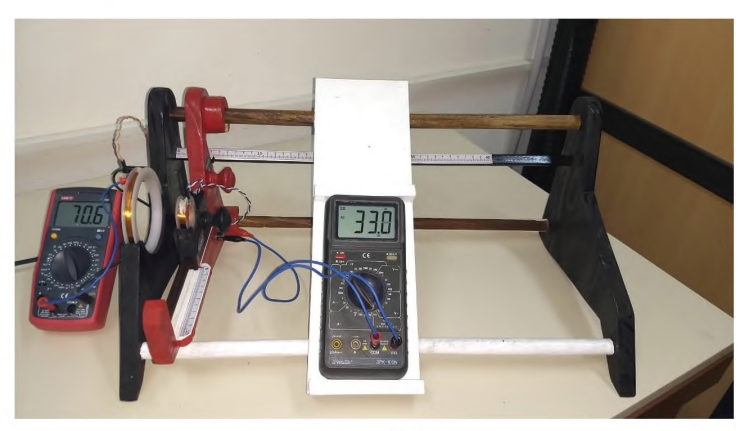

(b)

Figura 4: Fotografía del dispositivo durante la medición, en perspectiva (a) y de frente (b)

\section{Experimento}

Con el dispositivo construido en el laboratorio se obtienen los valores expuestos en las Figuras 5a y 6a para la f.e.m. inducida en la bobina secundaria B2, cuando el eje de ésta es respectivamente paralelo y perpendicular a la bobina primaria B1. Las tablas correspondientes se muestran en el Apéndice B.
Estas deben ser comparadas respectivamente con las Figuras 5b y $6 \mathrm{~b}$ obtenidas a partir del desarrollo expuesto en el Apéndice A. En ambos casos se aprecia que las tendencias son similares.

Cabe aclarar que B1 se encuentra con su centro en el origen de coordenadas y contenida en el plano (r,f.e.m.) o bien para el cálculo teórico del campo en el plano $(r, B)$. 


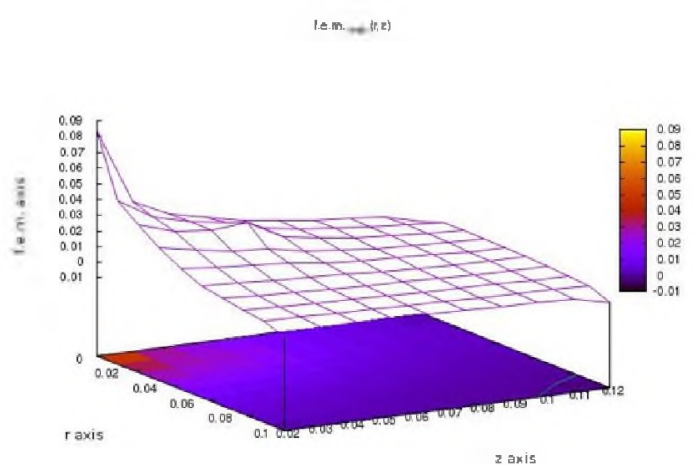

(a)

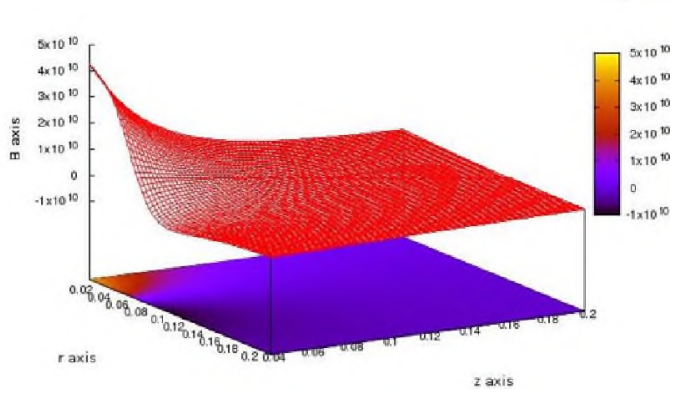

(b)

Figura 5: a) Valores experimentales de la fe.m. inducida en $\boldsymbol{B}_{2}$ [Volt] y b) Cálculos según las ecuaciones del Apéndice A del campo magnético axial $B_{2}[T]$.

Ambos en el rango $0.0 \mathrm{~cm}<\mathrm{r}^{\circ}<10.0 \mathrm{~cm}$ y $2.0 \mathrm{~cm}<\mathbf{z}<12.0 \mathrm{~cm}$ ctando $\boldsymbol{B}_{2}$ tiene stl eje perpendicular al de $\boldsymbol{B}_{t}$ Además Bi se dispone con centro en el origen de coordenadas y se encuentra contenida en el plano (r.fe.m. o bien $(\boldsymbol{r}, \boldsymbol{B})$.

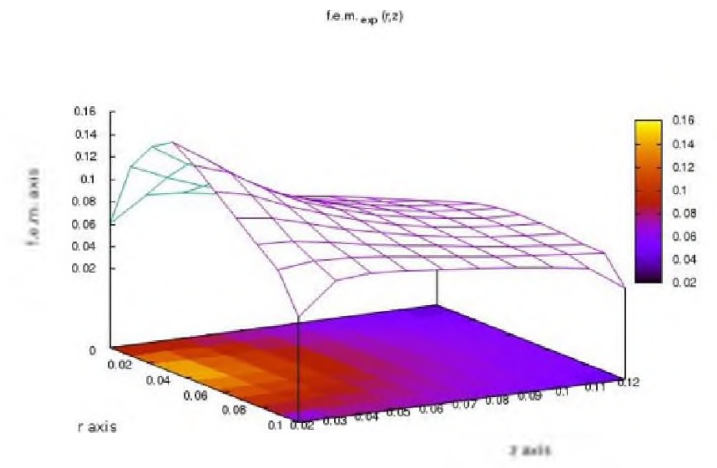

(a)

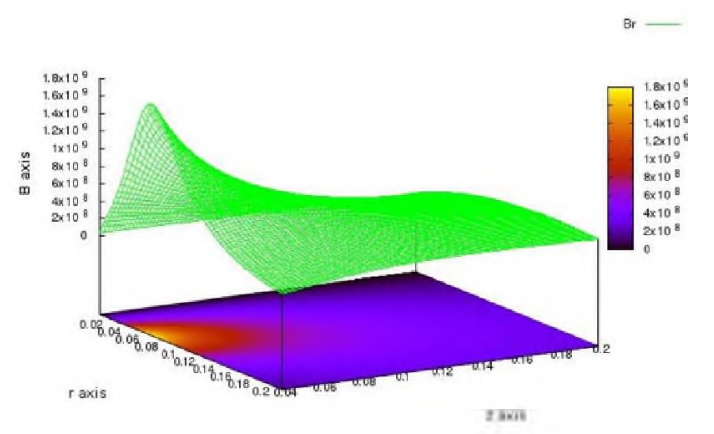

(b)

Figura 6: a) Valores experinentales de la fe.m. inducida en $B_{2}$ [Volt] y b) Cáculos segin las ecuaciones del apéndice $A$ del campo magnético axial $\left.\boldsymbol{B}_{3} / T\right]$.

Ambos en el rango $0.0 \mathrm{~cm}<r<10.0 \mathrm{~cm} y 2.0 \mathrm{~cm}<z<12.0 \mathrm{~cm}$, cuando $B_{2}$ tiene su eje perpendicular al de $B_{I}$ Además $B_{1}$ se dispone con centro en el origen de coordenadas y se encuentra contenida en el piano ( $r$. .e.m.) o bien $\left(r_{a} B\right)$.

\section{Conclusiones}

En este trabajo se propone el diseño de un dispositivo de construcción sencilla y económica, que resulta útil para el sen- sado del campo magnético producido por una espira circular en sus inmediaciones radiales $(\mathbf{r})$ y axiales $(\mathbf{z})$.

De la comparación entre las mediciones experimentales de la f.e.m. inducida y 
los valores calculados, se obtuvo un ajuste altamente satisfactorio; comprobando asî el funcionamiento eficaz del dispositivo diseñado.

A partir de la versatilidad del dispositivo desarrollado, se plantean algunas futuras aplicaciones con el fin de ahondar pedagógicamente en la temática, entre las que se incluye la comprobación fehaciente de la validez de la aproximación dipolar, la automatización de las mediciones mediante una placa Arduino y/o Raspberry que permita la lectura directa del campo magnético, o también una mayor precisión en la lectura de la f.e.m. inducida. Por otro lado consideramos que en el dispositivo actual, la comprobación de los mecanismos intervinientes en la transformación de las unidades de las medidas [V] a las utilizadas en los cálculos calculados [T] o viceversa, es un desafío bastante interesante para el estudiante.

Además, se asume que es posible alcanzar una mejor precisión de los datos obtenidos adquiriendo un multímetro de mayor sensibilidad y generando el campo magnético con la ayuda de una fuente alterna estabilizada.

\section{Agradecimientos}

Este trabajo fue realizado gracias a la financiación recibida a través del Proyecto PI 17/F008 Res 0966/17 CS. de la Secretaría General de Ciencia y Técnica (SGCyT) de la Universidad Nacional del Nordeste (UNNE).

\section{Bibliografía}

[1] Q. Jiang, X. Yang Zhou, J. Yao Chin and T. Jun Cui, Design and realization of a two-dimensional spatial magnetic field mapping apparatus to measure magnetic fields of metamaterials, J. App. Phys. 110 (2011) 024903.

[2] D. J. Griffiths, Introduction to Electrodynamics, 3rd. Ed. 1999 Prentice-Hall, Upper Saddle River, NJ, problema 5.48

[3] J. D. Jackson, Classical Electrodynamics, 3rd edn 1999 Wiley, New York, p 181-184.

[4] R. H. Jackson, Off-Axis Expansion Solution of Laplace's Equation: Application to Accurate and Rapid Calculation of Coil Magnetic Fields, IEEE Trans. Elect. Devices, 46, 1999, 1050-1062.

[5] R. H. Good, Elliptic integrals, the forgotten functions, Eur. J. Phys. 22 (2001) 119-126.

[6] http://www.sc.ehu.es/sbweb/fisica/elecmagnet/ campo_magnetico/espira/espira.html, consultada el 14 de Agosto de 2018.

[7] https://es.wikipedia.org/wiki/Integral_ el\%C3\%ADptica, consultada el 14 de Agosto de 2018.

[8] J. C. Simpson, J. E. Lane, C. D. Immer and R. C. Youngquist, Simple Analytic Expressions for the Magnetic Field of a Circular $\mathrm{Cu}^{-}$ rrent Loop, NASA Technical Reports, (2001) ID:20140002333, NASA/TM-2013-217919.

[9] M. Abramowitz, I. A. Stegun, Handbook of Mathematical Functions, Dover, 1972, p. 590.

[10] M. W. Garrett, Calculation of Fields, Forces, and Mutual Inductances of Current Systems by Elliptic Integrals, Journal of Applied Physics, 34(9), September 1963, p. 2567-2573.

[11] M. R. Spiegel y L. Abellanas Rapun, Fórmula y Tablas de Matemática Aplicada, 1988, McGrawHill, Madrid, p. 185. 


\section{Apéndice A: Cálculo del campo magnético de una espira circular en el espacio}

Calculamos el campo magnético producido por una espira circular en un punto fuera de su eje. Por la ley de Biot-Sarart se afirma que el campo B producido por una corriente î es $[2,3]$

$$
B=\frac{\mu_{0} I}{4 \pi} \int \frac{\overrightarrow{u_{t}} \times \overrightarrow{u_{r}}}{r^{2}} d l
$$

Siendo $d r$ el elemento de corriente, $\mathbf{u}_{t}$ es el vector unitario que señala la dirección y sentido de la

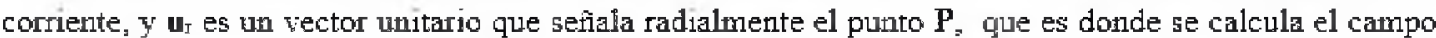
magnético.

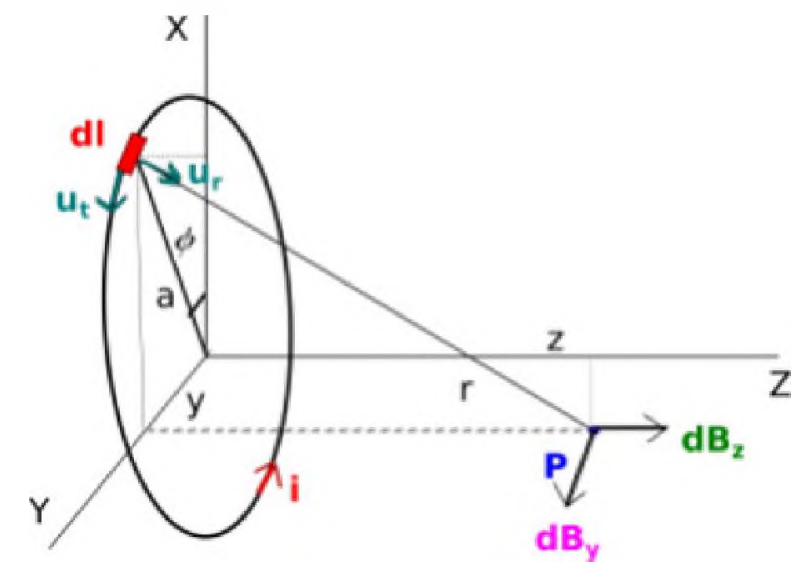

Figura 7: Esquena de la bobina $B_{2}$ y el canpo magnetico generado por ella

El problema planteado tiene simetria axial de forma que es suficiente calcular las componentes $B y$ y $B z$ del campo magnético en un punto $P(0, y, z)$ del platio $\mathbf{Y Z}$.

Como remos en la figura la distancia $\mathbf{r}$ entre el elemento de corriente $d l(d l=a \cdot d \rho) y P(0, y$, z), en el cual calculamos el campo magnético, es.

$$
r=\sqrt{a^{2}+y^{2}+z^{2}-2 a y \operatorname{sen} \phi}
$$

y los tersores se expresan como

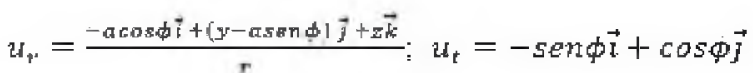

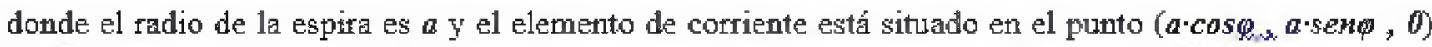
Haciendo el producto vectorial ut $*$ ur. las componentes del campo nagnético se escribirán

$$
B_{x}=\frac{\mu_{0}}{4 \pi} I \cdot a \cdot z \int_{0}^{2 \pi} \frac{\cos \phi}{r^{3}} d \phi_{;}^{-} \quad B_{y}=\frac{\mu_{0}}{4 \pi} I \cdot a \cdot z \int_{0}^{2 \pi} \frac{\sin \pi \phi}{r^{3}} d \phi ; \quad B_{z}=\frac{\mu_{0}}{4 \pi} I \cdot a \int_{0}^{2 \pi} \frac{(a-y \operatorname{sen} \phi)}{m^{3}} d \phi .
$$

La primera integral es inmediata y vale cero $(\boldsymbol{B x}=0)$, ya que $\int_{0}^{2 \pi} \cos \phi d \phi=0$ bien hablando en un sentido fisico para cada elemento de corriente $d l$ existe otro simétrico al plano $O Y Z$ cuyo efecto es el de 
anular la componente $X$ del campo magnético. De forma que las componentes del campo $B$ son solamente $B y$ (dirección radial) y $B z$ (a lo largo del eje de simetria).

Para el caso particular de $y=0$, que corresponde a un punto del eje de la espira, podemos comprobar fácilmente que $B y=0$ ya que $\int_{0}^{2 \pi} \operatorname{sen} \phi d \phi=0$ y además $=\sqrt{a^{2}+z^{2}}$ y $B_{z}=\frac{\mu_{0}}{2} \frac{l \cdot a}{r^{2}}$.

Por la simetría del problema también podemos reescribir estas dos contribuciones como sigue

$$
B_{y}=\frac{\mu_{0}}{2 \pi} I \cdot a \cdot z \int_{\frac{-\pi}{z}}^{\frac{\pi}{3}} \frac{\operatorname{sen} \phi}{r^{a}} d \phi: \quad B_{z}=\frac{\mu_{0}}{2 \pi} I \cdot a \int_{\frac{\pi}{z}}^{\frac{\pi}{2}} \frac{(a-y \operatorname{sen} \phi)}{r^{\pi}} d \phi .
$$

Para expresar estas integrales en términos de las integrales elipticas completas de primera y segunda especie se propone un cambio de variable $[5,6,7,8]$

$$
\theta=\frac{\pi}{2}-\phi \quad \Rightarrow \quad d \theta=-d \phi ; \quad \phi=\frac{\pi}{2} \Rightarrow \theta=0 ; \phi=\frac{-\pi}{2} \quad \Rightarrow \theta=\pi
$$

y la ecuación anterior para $B y$ queda

$B_{3^{\prime}}=\frac{\mu_{0}}{2 \pi} I \cdot a \cdot z \int_{\frac{J_{-\pi}^{2}}{2}}^{\frac{\pi}{2}} \frac{\operatorname{sen} \phi}{\left(a^{2}+y^{2}+z^{2}-2 a y \operatorname{sen} \phi\right)^{\frac{3}{2}}} d \phi=\frac{\mu_{0}}{2 \pi} I \cdot a \cdot z \int_{4 \pi}^{0} \frac{\cos \theta}{\left(a^{2}+y^{2}+z^{2}-2 a y \cos \theta\right)^{\frac{\pi}{2}}}(-d \theta)$

Llamando $\frac{a^{2}+y^{2}+z^{2}}{2 a y}=k(y, z)$ y $\frac{\mu_{0}}{2 \pi} \frac{1 a}{(2 a)^{\frac{3}{2}}}=C$ podemos reescribir la expresión anterior como

$$
B_{y}=C \cdot \frac{z}{y^{\frac{3}{2}}} \int_{0}^{\pi} \frac{\cos \theta}{(k(y, z)-\cos \theta)^{\frac{3}{2}}} d \theta
$$

Similarmente para la componente $B_{z}$ tenemos

$$
B_{z}=\frac{\mu_{0}}{2 \pi} I \cdot a \cdot \frac{\int^{\frac{\pi}{3}}}{\frac{\pi}{3}} \frac{(a-y \operatorname{sen} \phi)}{\left(a^{2}+y^{2}+z^{2}-2 a y^{2} \operatorname{sen} \phi\right)^{\frac{3}{2}}} d \phi=\frac{\mu_{0}}{2 \pi} I \cdot a \int_{\pi \pi}^{\pi} \frac{(a-y \cos \theta)}{\left(a^{2}+y^{2}+z^{2}-2 a y \cos \theta\right)^{\frac{2}{2}}}(-d \theta)
$$

la cual se reduce a

$$
B_{z}=C \frac{1}{y^{\frac{3}{2}}} \int_{0}^{\pi} \frac{(a-y \cos \theta)}{(k(y, z)-\cos \theta)^{\frac{3}{2}}} d \theta
$$

En ambos casos las integrales son elipticas y podemos resolverlas numéricamente en forma aproximada. Para ello recurrimos a las tablas $[4,5,6,7,8,9,10,11]$ obteniendo

$$
\int_{0}^{\pi} \frac{d \theta}{(R(y, I)-\cos \theta)^{\frac{3}{2}}}=\frac{m}{2-2 m} \sqrt{2 m} E(m) ; \int_{0}^{\pi} \frac{-\cos \theta d \theta}{(R(y, E)-\cos \theta)^{\frac{3}{2}}}=\sqrt{2 m} \mathcal{K}^{*}(m)-\frac{2-m}{2-2 m} \sqrt{2 m} E(m)
$$

Proponiendo la sustitución $m=\frac{2}{1+k} \Rightarrow k=\frac{2}{m}-1=\frac{2-m}{m}=$ podemos escribir:

- Integral eliptica completa de primera especie 


$$
K(m)=\int_{0}^{\frac{\pi}{2}} \frac{d \theta}{\sqrt{1-m^{2} \operatorname{sen}^{2} \theta}}=\frac{\pi}{2}\left|1+\left(\frac{1}{2}\right)^{2} m^{2}+\left(\frac{1 \cdot 3}{2 \cdot 4}\right)^{2} m^{4}+\left(\frac{1 \cdot 3 \cdot 5}{2 \cdot 4 \cdot 6}\right)^{2} m^{6}+\cdots+\left(\frac{(2 n-1) ! !}{2 n ! !}\right)^{2} m^{2 n}+\cdots\right|
$$

- Integral eliptica completa de segunda especie

$$
E(m)=\int_{0}^{\frac{\pi}{3}} \sqrt{1-m^{2} \operatorname{sen}^{2} \theta} d \theta=\frac{\pi}{2}\left[1-\left(\frac{1}{2}\right)^{2} m^{2}-\left(\frac{1 \cdot 3}{2 \cdot}\right)^{2} \frac{m^{4}}{3}-\left(\frac{1 \cdot 3 \cdot 5}{2 \cdot 4 \cdot 6}\right)^{2} \frac{m^{6}}{5}-\cdots-\left(\frac{(2 n-1) !}{2 n !}\right)^{2} \frac{m^{2 n}}{2 n-1}+\cdots\right]
$$

Consecuentemente, las componentes del campo magnético se plueden expresar en términos de las integrales elipticas completas de primera, $K(m)$, y segunda. $E(m)$, especie de la siguiente forma

$$
\begin{gathered}
B_{y}=c \cdot \frac{z}{y^{\frac{3}{2}}}\left[-\sqrt{2 m K(m)}+\frac{2-m}{2-2 m} \sqrt{2 m} E(m)\right] \\
B_{z}=c \cdot \frac{1}{y^{\frac{3}{2}}}\left[a \frac{m}{2-2 m} \sqrt{2 m} E(m)+y \sqrt{2 m} K(m)-y \frac{2-m}{2-2 m} \sqrt{2 m} E(m)\right]
\end{gathered}
$$

El resultado de graficar estas dos ecuaciones en el espacio se exponen en la Figura 8.

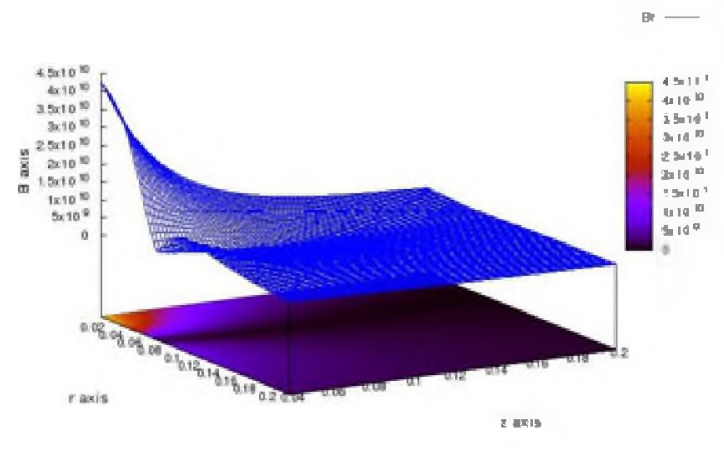

(a)

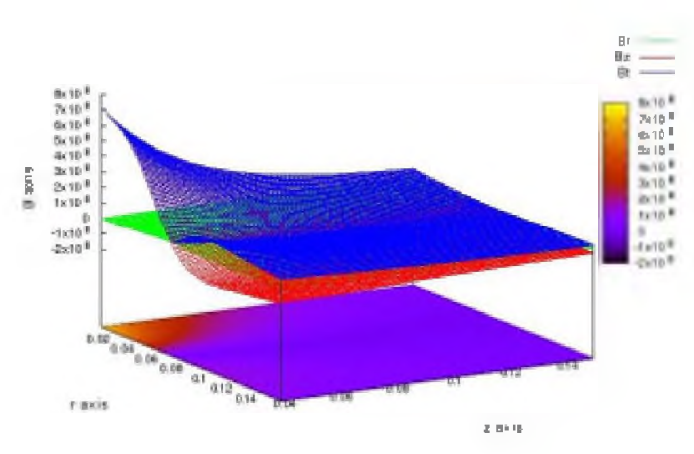

(b)

Figura 8: Calculo del campo magnético en $[T]$ al $B_{\text {toil }}$ en forma ass lada y bj junto a las contribuciones radial ty arial $z$ 


\section{Apéndice B: Valores medidos en el experimento}

Las caracteristicas del equipo constuido se especifican en la Tabla 1 y los valores medidos se especifican en las Tabla 2 y Tabla 3.

\begin{tabular}{|c|c|c|}
\hline \multicolumn{3}{|c|}{$\mathrm{BOB \Pi NA}\left(\mathrm{B}_{1}\right)$} \\
\hline Característica & Cantidad & Unidad \\
\hline$N_{1}$ & 1200 & Wueltas \\
\hline$I_{1}$ & $0.0657 \pm 0.001$ & A \\
\hline Radiol & $0.0475 \pm 0.001$ & $\mathrm{tm}$ \\
\hline$F_{1}$ & $10.36 \pm 0.01$ & $T$ \\
\hline \multicolumn{3}{|c|}{$\operatorname{BOBINA}\left(\mathbf{B}_{2}\right)$} \\
\hline Característica & Cantidad & Unidad \\
\hline $\mathrm{N}_{2}$ & 1200 & Vueltas \\
\hline Radion & $0.0155 \pm 0.01$ & m \\
\hline
\end{tabular}

Tabla 1: Caracterisficas de las bobinas del disposifivo de medicion

\begin{tabular}{|c|c|c|c|c|c|c|c|c|c|c|c|}
\hline \multirow[b]{3}{*}{ LOWGIUDTRAL } & \multicolumn{11}{|c|}{ CAMPO AXLAL (EJES PARALELOS) } \\
\hline & \multicolumn{11}{|c|}{ TRANSVERSAL } \\
\hline & 0.000 & 0.010 & 0.020 & 0.030 & 0.040 & 0.050 & 0.060 & 0.070 & $0.0 \$ 0$ & 0.090 & 0.100 \\
\hline 0.020 & 0.084 & 0.085 & 0.083 & 0.067 & 0.040 & 0.006 & 0001 & 0.006 & 0.003 & 0.001 & -0.001 \\
\hline 0.030 & 0.060 & 0.061 & 0.056 & 0.043 & 0.025 & 0.008 & -0.001 & -0.003 & -0.001 & -0.002 & -0.002 \\
\hline 0.040 & 0.041 & 0.041 & 0.035 & 0.027 & 0.016 & 0.007 & -0.004 & -0.006 & -0.005 & -0.004 & -0.004 \\
\hline 0.050 & 0.027 & 0.027 & 0.230 & 0.017 & 0.011 & 0.005 & 0.000 & -0.004 & -0.006 & -0.006 & -0.005 \\
\hline 0.060 & 0.019 & 0.018 & 0.016 & 0.012 & 0.007 & 0.003 & 0.000 & -0.003 & -0.005 & -0.006 & -0.006 \\
\hline 0.070 & 0.012 & 0.012 & 0.010 & 0.008 & 0.004 & 0.002 & -0.001 & -0.003 & -0.005 & -0.006 & -0.006 \\
\hline 0.080 & 0.007 & 0.007 & 0.005 & 0.004 & 0.002 & 0.000 & -0.001 & -0.003 & -0.004 & -0.005 & -0.006 \\
\hline 0.090 & 0.004 & 0.004 & 0.003 & 0.002 & 0.001 & -0.001 & -0.002 & -0.003 & -0.004 & -0.005 & -0.006 \\
\hline 0.100 & 0.002 & 0.002 & 0.001 & 0.000 & -0.001 & -0.002 & -0.003 & -0.004 & -0.005 & -0.005 & -0.006 \\
\hline 0.110 & 0.000 & 0.000 & -0.001 & -0.001 & -0.002 & -0.002 & -0.003 & -0.004 & -0.005 & -0.005 & -0.006 \\
\hline 0.120 & -0.001 & -0.002 & -0.002 & -0.002 & -0.003 & -0.003 & -0.004 & -0.004 & -0.005 & -0.005 & -0.006 \\
\hline
\end{tabular}

Tabla 2: Valores del campo magnético wial (ejes paralelos), medias en $V /$ en wha grila de Joxio cmo 


\begin{tabular}{|c|c|c|c|c|c|c|c|c|c|c|c|}
\hline & \multicolumn{11}{|c|}{ CAMPO TRANSVERSAL (EJES PERPENDICULARES) } \\
\hline & \multicolumn{11}{|c|}{ TRANSVERSAL } \\
\hline LONGITUDTAL & 0.000 & 0.010 & 0.020 & 0.030 & 0.040 & 0.050 & 0.060 & 0.070 & 0.080 & 0.090 & 0.100 \\
\hline 0.020 & 0.061 & 0.139 & 0.237 & 0.370 & 0.300 & 0.239 & 0.178 & 0.123 & 0.080 & 0.055 & 0.044 \\
\hline 0.030 & 0.056 & 0.107 & 0.190 & 0.268 & 0.299 & 0.240 & 0.177 & 0.122 & $0.0 \mathrm{~g} 7$ & 0.060 & 0.049 \\
\hline 0.040 & 0.050 & 0.088 & 0.139 & 0.178 & 0.187 & 0.165 & 0.140 & 0.110 & 0.084 & 0.065 & 0.051 \\
\hline 0.050 & 0.043 & 0.069 & 0.103 & 0.130 & 0.135 & 0.128 & 0.112 & 0.094 & 0.077 & 0.067 & 0.050 \\
\hline 0.060 & 0.037 & 0.055 & 0.077 & 0.094 & 0.098 & 0.099 & 0.092 & 0.082 & 0.067 & 0.065 & 0.048 \\
\hline 0.070 & 0.033 & 0.046 & 0.061 & 0.073 & 0.078 & 0.078 & 0.074 & 0.069 & 0.060 & 0.061 & 0.045 \\
\hline 0.090 & 0.030 & 0.039 & 0.051 & 0.059 & 0.062 & 0.064 & 0.062 & 0.059 & 0.053 & 0.057 & 0.042 \\
\hline 0.090 & 0.028 & 0.035 & 0.044 & 0.050 & 0.052 & 0.055 & 0.053 & 0.051 & 0.047 & 0.052 & 0.039 \\
\hline 0.100 & 0.026 & 0.031 & 0.038 & 0.043 & 0.045 & 0.047 & 0.045 & 0.045 & 0.041 & 0.049 & 0.036 \\
\hline 0.110 & 0.025 & 0.029 & 0.034 & 0.038 & 0.040 & 0.042 & 0.041 & 0.040 & 0.036 & 0.045 & 0.033 \\
\hline 0.120 & 0.025 & 0.027 & 0.031 & 0.034 & 0.036 & 0.037 & 0.037 & 0.036 & 0.032 & 0.042 & 0.031 \\
\hline
\end{tabular}

